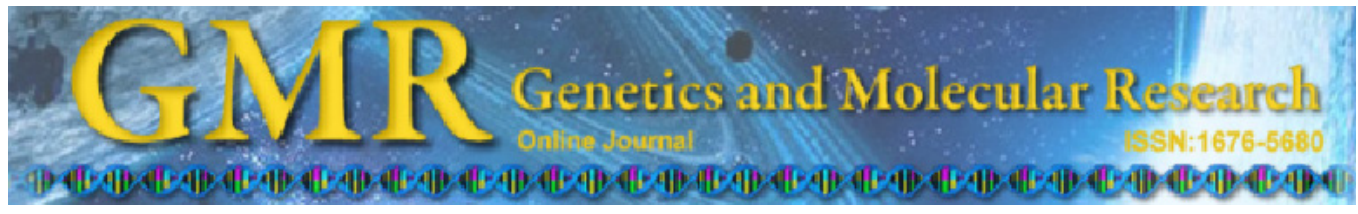

\title{
Improved method for predicting protein fold patterns with ensemble classifiers
}

\author{
W. Chen ${ }^{1}$, X. Liu ${ }^{1,3,4}$, Y. Huang', Y. Jiang ${ }^{1}$, Q. Zou' ${ }^{1}$ and C. Lin ${ }^{1}$ \\ ${ }^{1}$ School of Information Science and Technology, Xiamen University, \\ Xiamen, Fujian, China \\ ${ }^{2}$ Animal Science and Technology College, \\ Henan University of Science and Technology, Luoyang, Henan, China \\ ${ }^{3}$ Shenzhen Research Institute, Xiamen University, Shenzhen, \\ Guangzhou, China \\ ${ }^{4}$ Key Laboratory of Advanced Design and Intelligent Computing, \\ Dalian University, Ministry of Education, Dalian, China \\ Corresponding author: C. Lin \\ E-mail: chenlin@xmu.edu.cn
}

Genet. Mol. Res. 11 (1): 174-181 (2012)

Received August 5, 2011

Accepted January 6, 2012

Published January 27, 2012

DOI http://dx.doi.org/10.4238/2012.January.27.4

\begin{abstract}
Protein folding is recognized as a critical problem in the field of biophysics in the 21st century. Predicting protein-folding patterns is challenging due to the complex structure of proteins. In an attempt to solve this problem, we employed ensemble classifiers to improve prediction accuracy. In our experiments, 188-dimensional features were extracted based on the composition and physical-chemical property of proteins and 20-dimensional features were selected using a coupled position-specific scoring matrix. Compared with traditional prediction methods, these methods were superior in terms of prediction accuracy. The 188-dimensional feature-based method achieved 71.2\% accuracy in five cross-validations. The accuracy rose to $77 \%$ when we used a 20-dimensional feature vector. These methods were used on recent data, with $54.2 \%$ accuracy. Source codes and dataset, together with web server and software tools for prediction, are available at: http://datamining.xmu.edu.cn/main/ cwc/ProteinPredict.html.
\end{abstract}

Key words: Protein folding pattern; Ensemble classifier;

Machine learning; Bioinformatics 


\section{INTRODUCTION}

Proteomics has garnered more interest than genomics in the post-genomic era. Protein is an essential component in cells. Protein structure prediction and functional analysis are always the cores of bioinformatics research topics, and functional analysis always relies on structure prediction. However, it is difficult, costly, and not always feasible for all the proteins to obtain a tertiary structure through experimental means, and the accuracy of secondary structure prediction has, for a long time, been at a bottleneck. Folding pattern prediction falls somewhere in the middle of secondary structure prediction and tertiary structure prediction, which are directly related to function analysis. Therefore, increasing the accuracy of folding pattern prediction is essential. Studies have shown that the development of new drugs (Vendruscolo and Dobson, 2005), cancer research (Honda et al., 2005), and human immunodeficiency virus therapies (Boisvert et al., 2008) all require accurate prediction of associated protein folding patterns.

Although nuclear magnetic resonance is a powerful technique for determining membrane protein structures (Call et al., 2006; Douglas et al., 2007; Schnell and Chou, 2008), it requires expensive equipment and as much or more time than other methods. Homology modeling techniques (Chou, 2004), which are intended to develop decent models and provide structural information in a timely manner, have been widely used in drug design (Gao et al., 2007; Li et al., 2007). A hurdle occurs, however, when the query protein has no homologous protein of known structure in the existing databases.

Shen and Chou (2006) resorted to a taxonomic approach with underpinning based on the assumption that the number of protein folds is limited. Their study established a prediction web server called PFP-Pred, but its overall success rate on a stringent benchmark dataset (Ding and Dubchak, 2001) was just 62\%. Two additional predictors have since been developed with success rates of $61.1 \%$ (Nanni, 2006) and 68.4\% (Chen and Kurgan, 2007). In 2009, a predictor called PFP-FunDSeqE (Shen and Chou, 2009) implemented an approach that combines functional domain and sequential evolution information through a fusion ensemble classifier; the success rate reached $70.5 \%$. However, the dimensionality of the feather space was relatively high. Ding and Dubchak (2001) proposed 125 feathers, and Shen and Chou (2006) proposed 283, but only 313 training samples and 385 testing samples were used in the experimental evaluation. Although it has relatively high accuracy, PFP-FunDSeqE is time-consuming and presents challenges when dealing with large amounts of data. To solve these problems, we propose two kinds of feature extraction methods, of which one is designed for accuracy and the other to work quickly and maintain robust performance. Then, the ensemble learning method is used to improve performance for multi-class classification problems.

\section{MATERIAL AND METHODS}

\section{Dataset}

We chose two datasets for the experiments. One was the latest data in the SCOP database (Murzin et al., 1995) and the other was the same dataset as that in the PFP-Pred and PFP-FunDSeqE databases, which contain 311 proteins for training and 383 proteins for testing. None of the proteins in the testing dataset has more than $35 \%$ sequence similarity to those in the training dataset. According to the SCOP database these proteins can be further 
categorized into the following 27-fold types: 1) globin-like, 2) cytochrome c, 3) DNA-binding 3-helical bundle, 4) 4-helical up-and-down bundle, 5) 4-helical cytokines, 6) EF-hand, 7) immunoglobulin-like, 8) cupredoxins, 9) viral coat and capsid proteins, 10) concanavalin Alike lectin/glucanases, 11) SH3-like barrel, 12) oligonucleotide/oligosaccharide-binding-fold, 13) $\beta$-trefoil, 14) trypsin-like serine proteases, 15) lipocalins, 16) triosephosphate isomerase barrel, 17) flavin adenine dinucleotide (also nicotinamide adenine dinucleotide-binding motif), 18) flavodoxin-like, 19) nicotinamide adenine dinucleotide phosphate-binding Rossmann fold, 20) P-loop, 21) thioredoxin-like, 22) ribonuclease H-like motif, 23) hydrolases, 24) periplasmic binding protein-like, 25) $\beta$-grasp, 26) ferredoxin-like, and 27) small inhibitors, toxins, and lectins. Of these fold types, types 1-6 belong to the $\alpha$ structural class, types $7-15$ to the $\beta$ class, types $16-24$ to the $\alpha / \beta$ class, and types $25-27$ to the $\alpha+\beta$ class.

\section{Feature extraction method}

Feature extraction methods for protein sequence, which are important in the machine learning method for protein or structure function prediction, can be divided into two categories: one is calculated from a position-specific scoring matrix (PSSM; Schaffer et al., 2001; $\mathrm{Pu}$ et al., 2007), and the other is extracted from amino acid composition-position or physicalchemical properties. Because PSSM needs to be computed from the Basic Local Alignment Search Tool (BLAST) result, it is more a time-consuming method than extraction from amino acid properties.

We used both feature extraction methods in our study. We extracted the same 188 composition-position and physical-chemical property features as those of SVMProt (Cai et al., 2003), including hydrophobicity, normalized van der Waals volume, polarity, polarizability, charge, surface tension, secondary structure, and solvent accessibility, which are proven to work well for protein function prediction. We also calculated 20 features based on a PSSM as follows.

For every site in the given protein sequence, we calculated the possibility of replacing it with 20 types of amino acids from the Swiss-Prot database. We performed a BLAST analysis (blastpgp program) and put the resulting sequence into the Swiss-Prot database to get the PSSM. Then, we computed the average replaced possibility for all 20 amino acids and for the final 20 features.

\section{Ensemble classification method}

Studies have shown that choosing the appropriate ensemble classifiers can improve the accuracy of prediction in weak classification problems. In addition, the base classifier differentiation also helps to improve the performance of voting systems. We demonstrate an ensemble with two base classifiers that performs better in individual prediction. Because protein fold pattern prediction is a multi-class classification problem, the final result depends on the sum of the probabilities of the individual base classifiers.

Given the special nature of the data, we used various classifiers to achieve better results. We selected logistic model trees (LMT; Niels et al., 2005) and random forests (RF; Breimin, 2001) as base classifiers in 188-dimensional (D) experiments. For the 20-D experiments, we selected SimpleLogistic (Sumner et al., 2005) and RF. 
In ensemble classifiers in general, binary classification can use the vote strategy to predict the final result. However, this strategy is unsuitable for multi-class classification. In this study, we used the strategy of probability weighting. Each basic classifier predicts the test sample and outputs the probability of each class. The final result of the ensemble classifier indicates the class with the highest sum of probability. The flow is shown in Figure 1.

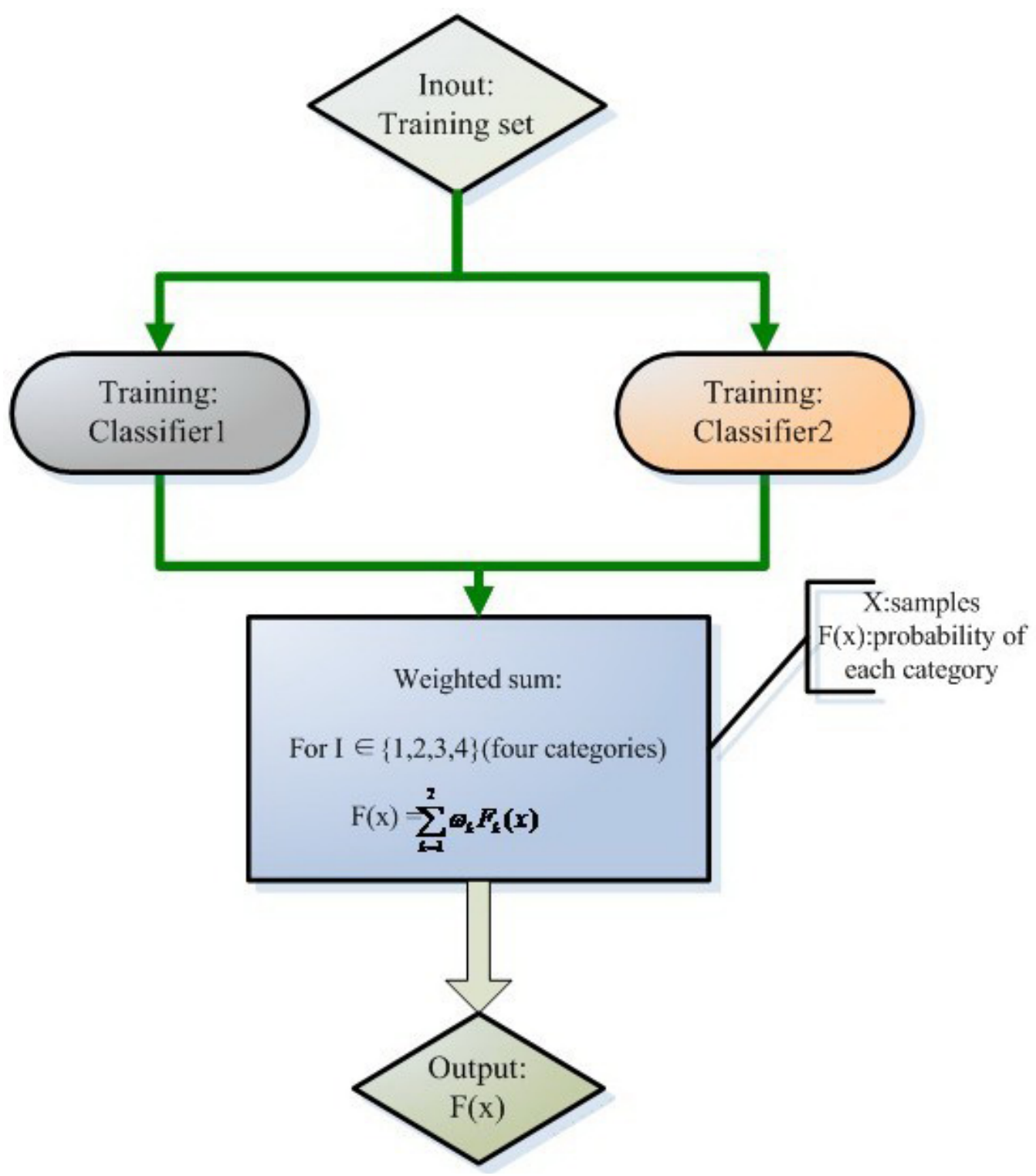

Figure 1. Multi-class ensemble strategy. 


\section{RESULTS}

\section{Comparison with related studies}

Many researchers have published related studies, and we performed tests on the same benchmark dataset (Nanni, 2006; Chen and Kurgan, 2007; Shen and Chou, 2006, 2009). The highest prediction accuracy rate of the other studies is only $70.5 \%$ (Shen and Chou, 2009). In our study, the accuracy of the 188 -D feature was $71.2 \%$ and rose to $77 \%$ when the PSSM method was used. This rate is much higher than that reported in other studies. The comparison is shown in Figure 2.

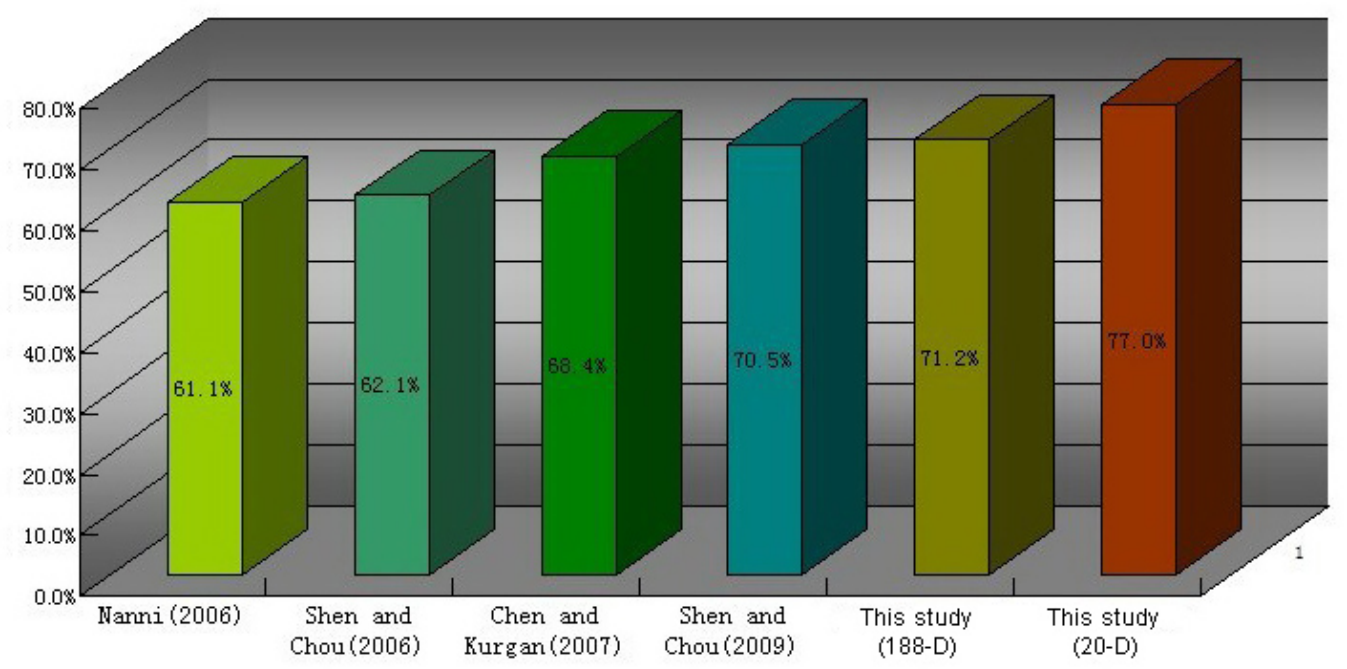

Figure 2. Comparison of six protein fold pattern recognition methods.

First, we extracted 188-D features for every protein in our dataset, including the training and testing sets; then, we predicted the whole dataset with each classifier using 5-fold cross-validation. The classifiers that performed well were chosen to build our ensemble classifier. Our previous experiments had shown that naive Bayes, RF, SimpleLogistic, and LMT have a relatively high accuracy in our prediction. We tried 2 and 3 classifiers to compose an ensemble classifier. We achieved the highest accuracy when LMT and RF were chosen as basic classifiers; accuracy of the 2-classifier ensemble classifier reached $71.2 \%$. Meanwhile, the 3 -classifier ensemble classifier performed at an accuracy of no more than $70.5 \%$. Hence, the ensemble classifier of LMT and RF outperformed others in our experiment, and it also worked well with regard to time and efficiency.

For the 20-D features, we extracted features for each sequences and used a method similar to that outlined above with 5-fold cross-validation to select basic classifiers for the ensemble. In this experiment, functional trees (FT), RF, SimpleLogistic, and LMT performed better than did the others. In our test of the 2-classifier ensemble classifier, the ensemble clas- 
sifier consisted of SimpleLogistic and RF and reached 77\% accuracy, which was an improvement compared with that of the 188-D features. The best accuracy for the 3-classifier ensemble classifier was $75.9 \%$.

\section{Performance on different classifiers}

Compared with other studies, the performance of our features proved their validity. Therefore, we tried to prove the superiority of our ensemble classifier. To show that the selected base classifiers in the classification are good choices, we performed a 5 -fold cross-validation on the entire dataset with different classifiers. Their performance on 4-fold patterns with the same 188-D features is shown in Table 1, whereas the performance with 20-D features is shown in Table 2. We compared our ensemble classifier with commonly used classifiers, including RF, decision tree (J48), naive Bayes, and K nearest neighbor (IB1), among others. Our experiments showed that the establishment of the ensemble classifiers was accurate and effective.

\begin{tabular}{|c|c|c|c|c|c|c|c|c|c|c|c|c|}
\hline \multirow[t]{2}{*}{ Classifiers } & \multicolumn{3}{|c|}{ all- $\alpha$} & \multicolumn{3}{|c|}{ all- $\beta$} & \multicolumn{3}{|c|}{$\alpha / \beta$} & \multicolumn{3}{|c|}{$\alpha+\beta$} \\
\hline & Precision & Recall & AUC & Precision & Recall & AUC & Precision & Recall & AUC & Precision & Recall & AUC \\
\hline SMO & 0.604 & 0.593 & 0.795 & 0.703 & 0.651 & 0.789 & 0.725 & 0.826 & 0.848 & 0.654 & 0.515 & 0.852 \\
\hline SimpleLogistic & 0.659 & 0.537 & 0.859 & 0.736 & 0.716 & 0.844 & 0.748 & 0.852 & 0.893 & 0.633 & 0.576 & 0.895 \\
\hline $\mathrm{RF}$ & 0.636 & 0.519 & 0.803 & 0.556 & 0.679 & 0.726 & 0.711 & 0.748 & 0.857 & 0.625 & 0.627 & 0.795 \\
\hline $\mathrm{J} 48$ & 0.511 & 0.444 & 0.695 & 0.512 & 0.578 & 0.668 & 0.658 & 0.652 & 0.76 & 0.593 & 0.485 & 0.792 \\
\hline NB & 0.4 & 0.333 & 0.702 & 0.646 & 0.468 & 0.757 & 0.616 & 0.809 & 0.869 & 0.5 & 0.545 & 0.859 \\
\hline IB1 & 0.545 & 0.222 & 0.592 & 0.634 & 0.413 & 0.642 & 0.49 & 0.896 & 0.675 & 0.625 & 0.152 & 0.57 \\
\hline Our ensemble classifier & 0.737 & 0.519 & 0.74 & 0.726 & 0.78 & 0.811 & 0.741 & 0.87 & 0.845 & 0.81 & 0.515 & 0.75 \\
\hline
\end{tabular}

$\mathrm{SMO}=$ sequential minimal optimization; $\mathrm{RF}=$ random forests; J48 = decision tree; $\mathrm{NB}=$ naive Bayes; IB1 $=\mathrm{K}$ nearest neighbor.

Table 2. Performance of different classifiers on protein fold pattern recognition (20-D features).

\begin{tabular}{|c|c|c|c|c|c|c|c|c|c|c|c|c|}
\hline \multirow[t]{2}{*}{ Classifiers } & \multicolumn{3}{|c|}{ all- $\alpha$} & \multicolumn{3}{|c|}{ all- $\beta$} & \multicolumn{3}{|c|}{$\alpha / \beta$} & \multicolumn{3}{|c|}{$\alpha+\beta$} \\
\hline & Precision & Recall & AUC & Precision & Recall & AUC & Precision & Recall & AUC & Precision & Recall & AUC \\
\hline SMO & 0.857 & 0.556 & 0.88 & 0.682 & 0.826 & 0.829 & 0.707 & 0.817 & 0.826 & 1 & 0.333 & 0.734 \\
\hline SimpleLogistic & 0.848 & 0.722 & 0.927 & 0.746 & 0.807 & 0.911 & 0.754 & 0.852 & 0.909 & 0.882 & 0.455 & 0.805 \\
\hline $\mathrm{RF}$ & 0.667 & 0.556 & 0.815 & 0.68 & 0.798 & 0.863 & 0.746 & 0.791 & 0.895 & 0.875 & 0.424 & 0.571 \\
\hline $\mathrm{J} 48$ & 0.423 & 0.407 & 0.626 & 0.667 & 0.642 & 0.746 & 0.664 & 0.704 & 0.762 & 0.531 & 0.515 & 0.761 \\
\hline NB & 0.773 & 0.315 & 0.789 & 0.523 & 0.716 & 0.745 & 0.646 & 0.461 & 0.791 & 0.345 & 0.606 & 0.768 \\
\hline IB1 & 0.806 & 0.463 & 0.72 & 0.686 & 0.661 & 0.749 & 0.662 & 0.852 & 0.799 & 0.778 & 0.636 & 0.807 \\
\hline Our ensemble classifier & 0.841 & 0.685 & 0.829 & 0.728 & 0.761 & 0.804 & 0.73 & 0.87 & 0.84 & 0.938 & 0.455 & 0.725 \\
\hline
\end{tabular}

For abbreviations, see legend to Table 1.

\section{Performance on the latest data}

We updated the data and retested our method. The former dataset has 311 proteins in the training dataset and 383 proteins in the testing dataset. The latest data contain $1067 \alpha$ structural proteins, $1034 \beta$ structural proteins, $1471 \alpha / \beta$ proteins, and $1588 \alpha+\beta$ proteins (data from SCOP, August 20, 2011). Some protein sequences were not found in the Universal Pro- 
tein Database, so they were removed, leaving $711 \alpha$ structural proteins, $695 \beta$ proteins, $929 \alpha / \beta$ proteins, and $973 \alpha+\beta$ proteins in our training set $\Phi$.

$$
\Phi=\Phi_{\alpha} \cup \Phi_{\beta} \cup \Phi_{\alpha / \beta} \cup \Phi_{\alpha+\beta}
$$

We used the PSSM method to process the dataset, and classification via regression and sequential minimal optimization (SMO) was then selected to combine the ensemble classifier. The final accuracy reached $54.2 \%$ with 5 -fold cross-validation.

\section{DISCUSSION}

We extracted 20- and 188-D features from protein sequences using composition-position and physical-chemical properties. The performance of the 20-D features was more accurate, whereas the extraction of the 188-D features was much faster. The results show that our method is feasible and greatly improves the efficiency of prediction.

An ensemble classifier is used to solve the protein fold recognition. Experiments show that this method achieved very good results. Compared with other identification methods, the ensemble classifier has many advantages, including short running time, features coming from only the test sequence, and better accuracy. Meanwhile, we have developed an online server and a software tool that can be downloaded and run on a personal computer. The open source software can be accessed for free at http://datamining.xmu.edu.cn/main/ cwc/ProteinPredict.html.

\section{ACKNOWLEDGMENTS}

Research supported by the Natural Science Foundation of China (\#61001013,\#61102136 and \#60971085), and the Natural Science Foundation of Fujian Province of China (\#2011J05158 and \#2010J01350), the Base Research Project of Shenzhen Bureau of Science, Technology and Information (\#JC201006030858A), the Opening Fund of Key Laboratory of Image Processing and Intelligent Control, Ministry of Education (\#200905), and the Opening Fund of Advanced Design and Intelligent Computing (Dalian University), Ministry of Education.

\section{REFERENCES}

Boisvert S, Marchand M, Laviolette F and Corbeil J (2008). HIV-1 coreceptor usage prediction without multiple alignments: an application of string kernels. Retrovirology 5: 110.

Breimin L (2001). Random forests. Machine Learn. 45: 5-32.

Cai CZ, Han LY, Ji ZL, Chen X, et al. (2003). SVM-Prot: Web-based support vector machine software for functional classification of a protein from its primary sequence. Nucleic Acids Res. 31: 3692-3697.

Call ME, Schnell JR, Xu C, Lutz RA, et al. (2006). The structure of the zetazeta transmembrane dimer reveals features essential for its assembly with the T cell receptor. Cell 127: 355-368.

Chen K and Kurgan L (2007). PFRES: protein fold classification by using evolutionary information and predicted secondary structure. Bioinformatics 23: 2843-2850.

Chou KC (2004). Structural bioinformatics and its impact to biomedical science. Curr. Med. Chem. 11: 2105-2134.

Ding CHQ and Dubchak I (2001). Multi-class protein fold recognition using support vector machines and neural networks. Bioinformatics 17: 349-358.

Douglas SM, Chou JJ and Shih WM (2007). DNA-nanotube-induced alignment of membrane proteins for NMR structure determination. Proc. Natl. Acad. Sci. U. S. A. 104: 6644-6648.

Gao WN, Wei DQ, Li Y, Gao H, et al. (2007). Agaritine and its derivatives are potential inhibitors against HIV proteases. 
Med. Chem. 3: 221-226.

Honda M, Kawai H, Shirota Y, Yamashita T, et al. (2005). cDNA microarray analysis of autoimmune hepatitis, primary biliary cirrhosis and consecutive disease manifestation. J. Autoimmun. 25: 133-140.

Li Y, Wei DQ, Gao WN, Gao H, et al. (2007). Computational approach to drug design for oxazolidinones as antibacterial agents. Med. Chem. 3: 576-582.

Murzin AG, Brenner SE, Hubbard T and Chothia C (1995). SCOP: a structural classification of proteins database for the investigation of sequences and structures. J. Mol. Biol. 247: 536-540.

Nanni L (2006). A novel ensemble of classifiers for protein fold recognition. Neurocomputing 69: 2434-2437.

Niels L, Mark H and Eibe F (2005). Logistic model trees. Machine Learn 95: 161-205.

$\mathrm{Pu} \mathrm{X}$, Guo J, Leung H and Lin Y (2007). Prediction of membrane protein types from sequences and position-specific scoring matrices. J. Theor. Biol. 247: 259-265.

Schaffer AA, Aravind L, Madden TL, Shavirin S, et al. (2001). Improving the accuracy of PSI-BLAST protein database searches with composition-based statistics and other refinements. Nucleic Acids Res. 29: 2994-3005.

Schnell JR and Chou JJ (2008). Structure and mechanism of the M2 proton channel of influenza A virus. Nature 451: 591-595.

Shen HB and Chou KC (2006). Ensemble classifier for protein fold pattern recognition. Bioinformatics 22: 1717-1722.

Shen HB and Chou KC (2009). Predicting protein fold pattern with functional domain and sequential evolution information. J. Theor. Biol. 256: 441-446.

Sumner M, Frank E and Hall MA (2005). Speeding up Logistic Model Tree Induction. In: Proceedings of 9th European Conference on Principles and Practice of Knowledge Discovery in Databases, Porto, Portugal (Jorge A, ed.). Springer, Germany, 675-683.

Vendruscolo M and Dobson CM (2005). A glimpse at the organization of the protein universe. PNAS 102: 5641-5642. 